\title{
Eventos de precipitación intensa en Paraguay: aspectos climáticos y estudio de casos
}

El conocimiento del comportamiento de la precipitación y las causas que desencadenan eventos de lluvias intensas es de extrema necesidad para permitir medidas ambientales apropiadas para mitigar las consecuencias negativas a la sociedad. El objetivo de este estudio es analizar climatológicamente la precipitación intensa diaria en Paraguay y las características sinópticas que influencian los eventos intensos en las localidades de Villarica (23/07/2014) y Capitán Meza (26/01/2002). Aquí, el análisis climático se realizó mediante la distribución de los eventos superiores al percentil 99 (denominados eventos de precipitación intensa) para la serie histórica entre 1999 a 2019 y para los sistemas sinópticos se realizó mediante análisis de campos meteorológicos utilizando datos de reanálisis del ERA5 e imágenes satelitales. En relación al comportamiento estacional de la precipitación, los eventos intensos se presentan más homogéneos durante todo el año en la región Oriental y en mayor número, en discrepancia con la región Occidental que se presentan más en verano y en primavera. Para los eventos sinópticos, se observó sistemas convectivos, manifestándose por medio de forzantes térmicas de bajos niveles, influenciado por el Jet de bajos niveles y por las forzantes dinámicas con la presencia de una vaguada de gran amplitud en altos niveles, siendo más visible para el caso de invierno.

Palabras clave: Eventos de lluvia intensa; Jet de bajos niveles; ERA5.

\section{Eventos de forte precipitação no Paraguai: aspectos climáticos e estudos de caso}

\begin{abstract}
O conhecimento do comportamento das precipitações e das causas que desencadeiam chuvas intensas é extremamente necessário para permitir medidas ambientais adequadas que mitiguem as consequências negativas para a sociedade. O objetivo deste estudo é analisar climatologicamente a intensa precipitação diária no Paraguai e as características sinóticas que influenciam os eventos intensos nas cidades de Villarica (23/07/2014) e Capitán Meza (26/01/2002). Aqui, a análise do clima foi realizada distribuindo os eventos acima do percentil 99 (chamados de eventos de precipitação intensa) para as séries históricas entre 1999 e 2019 e para os sistemas sinópticos foi realizada pela análise de campos meteorológicos usando dados de reanálise do ERA5 e imagens de satélite. Em relação ao comportamento sazonal da precipitação, os eventos intensos são mais homogêneos ao longo do ano na região Leste e em maior número, em discrepância com a região Oeste, que ocorrem mais no verão e na primavera. Para os eventos sinópticos, foram observados sistemas convectivos, manifestados por meio de forçantes térmicos de baixo nível, influenciados pelo Jet de baixo nível e por forçantes dinâmicos com presença de calha de grande amplitude em altos níveis, sendo mais visíveis no inverno.
\end{abstract}

Palavras-chave: Eventos de chuva forte; Jato de baixo nível; ERA5.

Topic: Meteorologia, Climatologia e Mudanças Climáticas

Reviewed anonymously in the process of blind peer.
Received: 04/03/2021

Approved: 23/03/2021

Nelva Bugoni Riquetti

Universidade Federal de Pelotas, Brasil

http://lattes.cnpq.br/1775726571682899

nelva.bugoni@gmail.com

André Becker Nunes (iD)

Universidade Federal de Pelotas, Brasil

http://lattes.cnpq.br/1873505066686878

http://orcid.org/0000-0002-4881-5810

andre.nunes@ufpel.edu.br

Referencing this:

RIQUETTI, N. B.; NUNES, A. B.. Eventos de precipitación intensa en Paraguay: aspectos climáticos y estudio de casos. Revista Ibero Americana de Ciências Ambientais, v.12, n.3, p.169-181, 2021. DOI: http://doi.org/10.6008/CBPC2179-6858.2021.003.0016 


\section{INTRODUCCIÓN}

El continente americano presenta climas tropicales, subtropicales y extra tropicales siendo influenciados por la temperatura del mar de los océanos adyacentes, por la Cordillera de los Andes y la Amazonía (SATYAMURTY et al., 1998). La precipitación experimenta gran variabilidad en América del Sur destacándose mayormente en otoño y en el verano austral, siendo el ENSO (EI Niño Southern Oscillation) la fuente más importante de modificación para todas las estaciones del año, excepto en el verano que es influenciado mayormente por los procesos locales de interacción superficie-atmósfera (GRIMM, 2003; 2011). En este sentido, estudios conducidos por Seluchi et al. (2012a) y Seluchi et al. (2012b), indican que en América del Sul el sistema sinóptico conocido como Baja del Noroeste Argentino (BNOA) influye en el tiempo en el centro-norte de Argentina, sur de Bolivia, Paraguay y las regiones sur y sureste, y en parte las regiones centrooeste y norte de Brasil, contribuyendo con las precipitaciones intensas.

La variabilidad climática existente en Paraguay es marcada entre regiones, lo que influye directamente en la economía del país, con fuerte dependencia de las actividades primarias (agricultura en larga escala y ganadería). En este sentido, según la clasificación climática de Köppen (PEEL et al., 2007), Paraguay posee tres climas predominantes, Tropical, Árido y Temperado, siendo Am (Monzónico) para la región Occidental, conjuntamente con BSh (Estepario cálido) y Cfa (Subtropical húmedo) para la región Oriental.

Los mayores índices de precipitación se observan al sur de Paraguay con precipitación homogénea a lo largo del año (1750-2100 mm/año), no obstante, para la región centro norte del país, la precipitación presenta comportamiento de máximas en verano y mínimas en invierno, con variación entre 700 y 1400 mm (REBOITA et al., 2010).

Las tendencias históricas en las últimas décadas sugieren aumento en la cantidad de eventos intensos de lluvia para la región Oriental del país. Esa tendencia positiva de la precipitación puede traer consecuencias negativas para la economía, especialmente por las pérdidas en la producción agropecuaria, problemas sociales y ambientales relacionados a inundaciones de ríos, problemas en áreas urbanas por lluvias intensas y por la falta de infraestructura, contaminación, perdida del hábitat entre otros, así como, pueden afectar negativamente la distribución temporal de las lluvias en el país que es dependiente directo del agronegocio.

En este contexto, el objetivo de estudio es documentar el comportamiento de las precipitaciones máximas diarias en Paraguay, especialmente en la región Oriental, donde se concentra en mayor parte los eventos intensos de precipitación, conjuntamente con el análisis sinóptico de los fenómenos que originan los casos más intensos de lluvia para las localidades de Villarica y Capitán Meza, apuntando reducir la resiliencia. Cabe recalcar, eventos intensos para Villarica y región en invierno son atípicos.

\section{MATERIALES Y MÉTODOS}

En este trabajo, se llevó a cabo el estudio de series históricas de datos de precipitación diaria, conjuntamente con el análisis sinóptico de 2 casos de eventos intensos de precipitación. Para el análisis 
climatológico se utilizaron series diarias de precipitación de 12 estaciones, administradas por la Dirección Nacional de Aeronáutica Civil (DINAC) del Paraguay en el periodo de 1999 a 2019. El criterio adoptado para seleccionar las estaciones fue el de no poseer fallas superiores al $2 \%$ en la serie histórica. Los eventos de precipitación intensa fueron identificados de acuerdo con los datos diarios acumulados aplicando el percentil 99 (p99). Posteriormente se verificó en cuales estaciones del año se encontraron los eventos p99.

Para el análisis sinóptico de los estudios de casos, fueron utilizados datos de reanálisis del ERA5 (HERSBACH et al., 2019) del ECMWF (European Center for Medium-Range Weather Forecast), con resolución horizontal de $30 \mathrm{~km}$, resolución temporal de 1 hora y 137 niveles verticales, con dominio espacial que cubre América del Sur y océanos adyacentes. Se utilizaron datos de presión a nivel medio del mar (pnmm), altura geopotencial, dirección y magnitud del viento, temperatura del aire y humedad específica para diferentes niveles verticales. También se generaron campos de la diferencia (llamados de ahora en adelante "campos de anomalía") entre el día del evento intenso y la normal climatológica (1980-2009) estacional. La nebulosidad asociada a los sistemas sinópticos es observada mediante el uso de imágenes de satélite (canal infrarrojo) obtenidas de la Divisão de Satélites e Sistemas Ambientais del Instituto Nacional de Pesquisas Espaciais (DSA/INPE) de Brasil.

\section{RESULTADOS Y DISCUSIÓN}

\section{Análisis climatológico}

A continuación (Tabla 1) se presentan las estaciones seleccionadas con sus respectivos valores de p99.

Tabla 1: Valores de p99 por cada estación meteorológica.

\begin{tabular}{lll}
\hline Región & Estaciones meteorológicas & Precipitación (p99) \\
\hline \hline \multirow{3}{*}{ Occidental } & Gral. Bruguez & 103,4 \\
\cline { 2 - 3 } Oriental & La Victoria & 111,2 \\
\cline { 2 - 3 } & Aeropuerto Guaraní & 91,8 \\
\hline & Aeropuerto Silvio Pettirossi & 96,5 \\
\hline & Caazapá & 62,0 \\
\cline { 2 - 3 } & Capitán Meza & 98,1 \\
\hline Concepción & 66,6 \\
\hline & Cnel. Oviedo & 69,0 \\
\hline & Pedro Juan Caballero & 83,6 \\
\hline Pilar & 115,3 \\
\cline { 2 - 2 } & San Estanislao & 66,6 \\
\cline { 2 - 2 } & Villarrica & 70,6 \\
\hline
\end{tabular}

Así, la mayoría de las estaciones operadas por DINAC están distribuidas en la región Oriental debido a los mayores índices pluviométricos en la región sur de Paraguay. En este sentido, en la Figura 1, se observa la localización de las estaciones meteorológicas, a la par los gráficos representando los eventos intensos de precipitación para verificar la distribución estacional de los días con eventos de lluvia intensa.

En relación a las estaciones localizadas en la región Occidental, estación La Victoria y Gral. Bruguez, no presentan eventos intensos en invierno (JJA), así también, para las demás estaciones del año presentan comportamiento semejante, a diferencia, en primavera se observaron más eventos intensos en La Victoria, 
por otra parte, en la estación Gral. Bruguez, sucedieron más eventos intensos, siendo el verano, la época con mayor cantidad de eventos intensos. Estos resultados concuerdan con lo presentado por Reboita et al. (2012) en donde describen que los eventos máximos de precipitación ocurren mayormente en verano y menos en invierno en la región centro-norte del Chaco paraguayo. Tal comportamiento se presenta principalmente a causa del mayor calentamiento superficial, favoreciendo el desenvolviendo de eventos convectivos en la región.

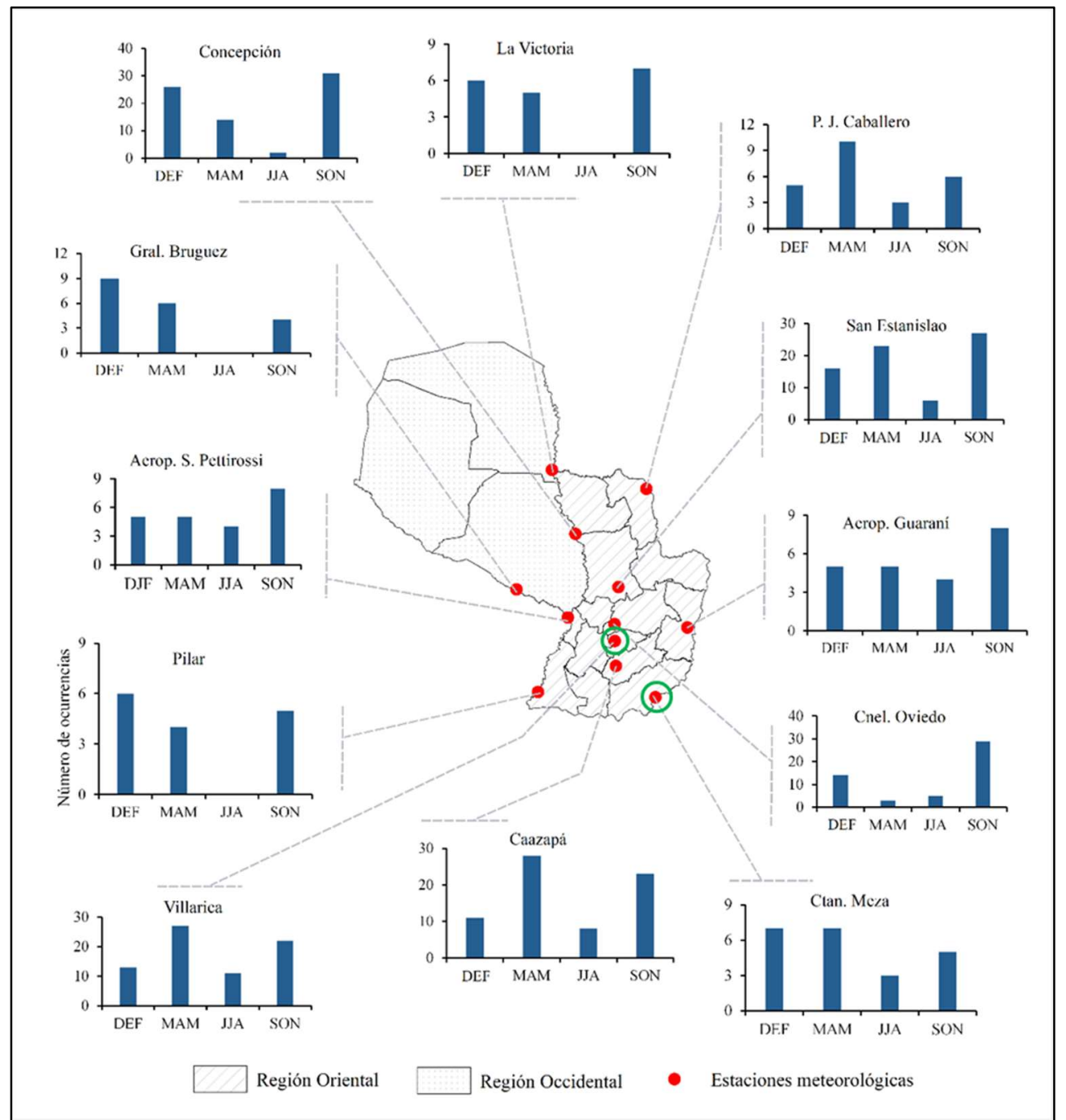

Figura 1: Localización de las estaciones meteorológicas en Paraguay, conjuntamente con gráficos, indicando la cantidad de eventos intensos de precipitación, distribuidos por cada estación del año. Diciembre, Enero, Febrero (DEF); Marzo, Abril, Mayo (MAM); Junio, Julio, Agosto (JJA); Septiembre, Octubre, Noviembre (SON). En contorno verde las ciudades con los casos analizados sinópticamente.

En este mismo orden de ideas, la distribución para la región Oriental, en la estación Pilar no mostraron eventos intensos para el invierno, en secuencia, Concepción, Cnel. Oviedo y San Estanislao para JJA presentan eventos intensos reducidos, a diferencia de MAM (otoño) en donde se observa padrón opuesto entre localidades, no obstante, presentan predominancia de eventos máximos precipitados en primavera (SON).

Posteriormente, para las estaciones que presentaron comportamiento más homogéneo, tenemos en la localidad del Aerop. Guaraní y Aerop. S. Pettirossi destacándose con mayor número de eventos en primavera, así también, la estación Capitán Meza, se pudo verificar la existencia de cierta semejanza entre 
verano y otoño, con cantidad mayor de eventos intensos para esas épocas del año. Por otra parte, en las estaciones Villarica y Caazapá, la predominancia de los eventos se dio en otoño y primavera, así como, para verano (DEF) e invierno, menos pronunciados.

En resumen, cuanto más al centro de la región Oriental, la ocurrencia de eventos intensos va en ascenso. Cabe enfatizar, las estaciones con mayores ocurrencias fueron Villarica, Concepción, Caazapá y San Estanislao (superior a 70 eventos), no obstante, localidades con ocurrencia de eventos inferiores a 20, se destacan las localidades Gral. Bruguez y La Victoria. Tal comportamiento se puede atribuir a las características climáticas que distinguen ambas regiones, concordando con el estudio de Bidegain et al. (2017) que cuanto más al norte de Paraguay la precipitación media anual disminuye con máximos de $600 \mathrm{~mm}$ en el Chaco paraguayo y 2000mm al sur, en la frontera con Paraná-Brasil, así también, el comportamiento es influenciado por los sistemas sinópticos típicos asociados a esas latitudes que contribuyen para eventos intensos de precipitación.

Existe relación entre la precipitación media y los casos intensos de precipitación, es decir, las regiones con mayor precipitación tienden a tener mayor número de eventos intensos. En este sentido, Mendonça et al. (2014) indican que la precipitación aumenta para latitudes mayores cuando la ZCIT se encuentra más al sur de la línea del Ecuador y cuando el sistema en altos niveles, conocido como Alta de Bolivia (AB) se encuentra más al norte de su posición normal. Estudios conducidos por Liebmann et al. (2004) y Haylock et al. (2006) evidencian aumento de la precipitación anual, en promedio de días lluviosos en Paraguay, especialmente entre EFM. En este sentido, Reboita et al. (2012) destacan para la región Sur de Paraguay y Brasil presenta un comportamiento más homogéneo con índices elevados de precipitación a lo largo del año, superior a 1700mm (REBOITA et al., 2010). En las regiones sur y sureste de Brasil (próximas a la región Oriental en Paraguay) en primavera la precipitación empieza a aumentar, estando influenciados por los sistemas frontales actuantes en esa región y por la Corriente en chorro subtropical en altos niveles (OLIVEIRA, 1986; VASQUES, 2007). Adicionalmente, Vasques (2007) destaca que en los meses de verano se presentan mayores índices precipitados y en invierno las menores amplitudes en la región sur de América del Sur. Para las estaciones más al sur de Paraguay es posible observar que el comportamiento de los eventos intensos se presentó más pronunciado en primavera y verano, concordando con los resultados presentado por Vasques (2007), no obstante, para las estaciones centro-norte y norte de la región Oriental los eventos se destacan en otoño.

Para estudiar la causa de los eventos intensos de lluvia se eligieron 2 eventos de precipitación para el análisis sinóptico en orden al mayor volumen precitado para las localidades elegidas, así como, en función de las diferentes estaciones del año para verificar las diferentes configuraciones sinópticas. En este sentido, las estaciones meteorológicas seleccionadas: Villarica (23/07/2014) y Capitán Meza (26/01/2002) con precipitación de $127,7 \mathrm{~mm} /$ día y $164 \mathrm{~mm}$ /día respectivamente. En la Figura 2 es posible observar la cantidad de eventos de precipitación intensa por estación del año.

En este contexto, se evidencia que la cantidad de eventos intensos ocurrió mayormente en la localidad de Villarica con 73, distribuidos en mayor número de ocurrencias para otoño, seguidos en la 
primavera, verano e invierno. Así también, en la estación Capitán Meza se presentaron 22 eventos, en donde se verifica homogeneidad para verano y otoño en cantidad de eventos, seguido en primavera e invierno. Cabe enfatizar, pese a la proximidad entre las localidades estudiadas (Figura 1) aun así proporciona diferencias entre número de eventos en cada ciudad.

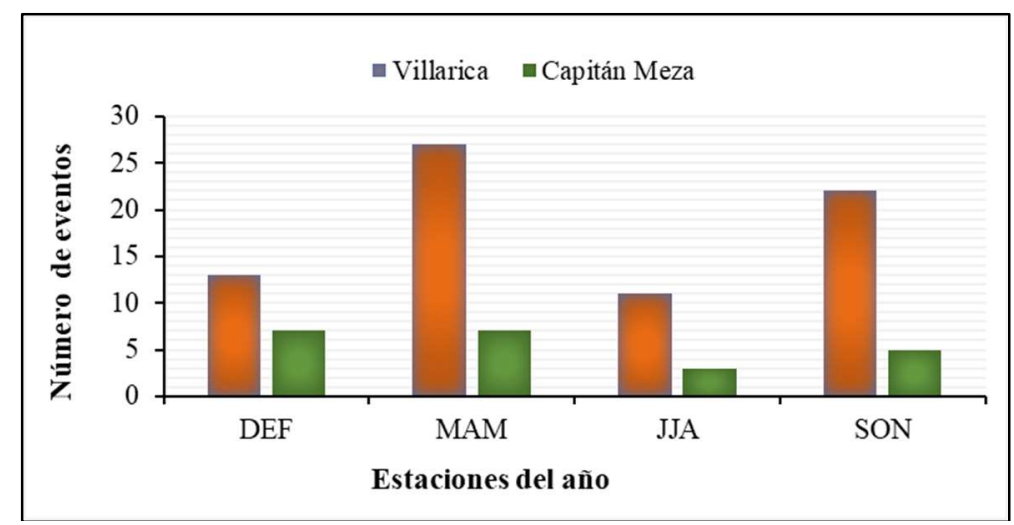

Figura 2: Distribución estacional de los eventos intensos de precipitación para la localidad de Villarica y Capitán Meza.

En otro orden de ideas, en la Figura 3, se observa el gráfico de dispersión y de tendencia de los eventos de lluvia intensa para las 2 localidades.

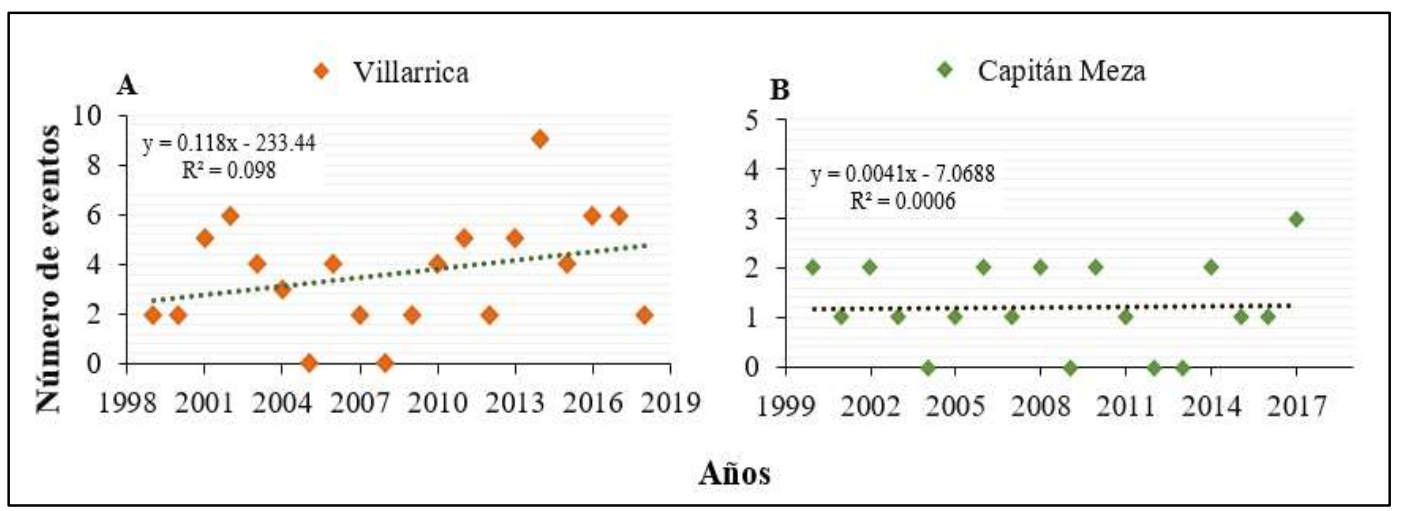

Figura 3: Gráfico de dispersión y recta de tendencia linear de los eventos p99 en el periodo entre 1998-2019 por estación meteorológica. A) Villarica; B) Capitán Meza.

La Figura 3 presenta tendencia positiva de los eventos p99 para las localidades de Villarica y Capitán Meza, esto implica que los eventos se han vuelto más frecuentes (aunque no estadísticamente significativo). Cabe destacar, la tendencia positiva se presenta más claramente en Villarica. La elección del filtro p99 favoreció a la baja significancia estadística por haber limitado el número de eventos, sin embargo, se eligió a causa de capturar los eventos más intensos de la serie histórica. No obstante, la variabilidad climática en consecuencia de la posible influencia del ENOS, tiende a contribuir para la baja significancia de la tendencia. A continuación, los resultados concuerdan con estudios llevados a cabo por Haylock et al. (2006) para la región sudeste de AS entre 1961 a 1990, sugiriendo tendencia positiva de eventos con lluvia intensa superior al p99, así también, Alexander et al. (2006) presentan resultados semejantes para la misma región con índices de precipitación extrema anual en el periodo de 1991-2000 para Paraguay, sur de Brasil y norte de Argentina. Bidegain et al. (2017) destacan la variabilidad interanual de la precipitación es fuertemente influenciada por el ENSO en la Cuenca del Río de la Plata, con tendencia positiva de la precipitación en gran parte de la cuenca, 
atribuida mayormente a eventos extremos de lluvias superiores al p99 y para el Chaco paraguayo muestran tendencia levemente negativa para el periodo entre 1971 a 2015.

Del mismo modo, los resultados encontrados en el estudio de eventos extremos de precipitación por Pastén et al. (2007) ubicadas en diferentes localidades de Paraguay en el periodo 1960 a 2004 muestran tendencia positiva de aumento de la precipitación total anual, en especial para las localidades de Villarica y Mariscal Estigarribia (localidad próxima a la estación Capitán Meza), sin embargo, tendencias negativas se presentan en Pilar y Pedro Juan Caballero. De igual forma, los estudios conducidos por Breuer et al. (2017) mostraron tendencia positiva en cantidad y frecuencia de eventos extremos en primavera, verano y otoño para Capitán Meza y para la estación del Aerop. Guaraní, aplicando el percentil 90 para el periodo 1976 a 2015, resultados coherentes con lo encontrado en este estudio.

\section{Análisis sinóptico}

En la Figura 4 se presenta los campos de pnmm para los dos casos de eventos más intensos de precipitación registrados en la serie histórica.

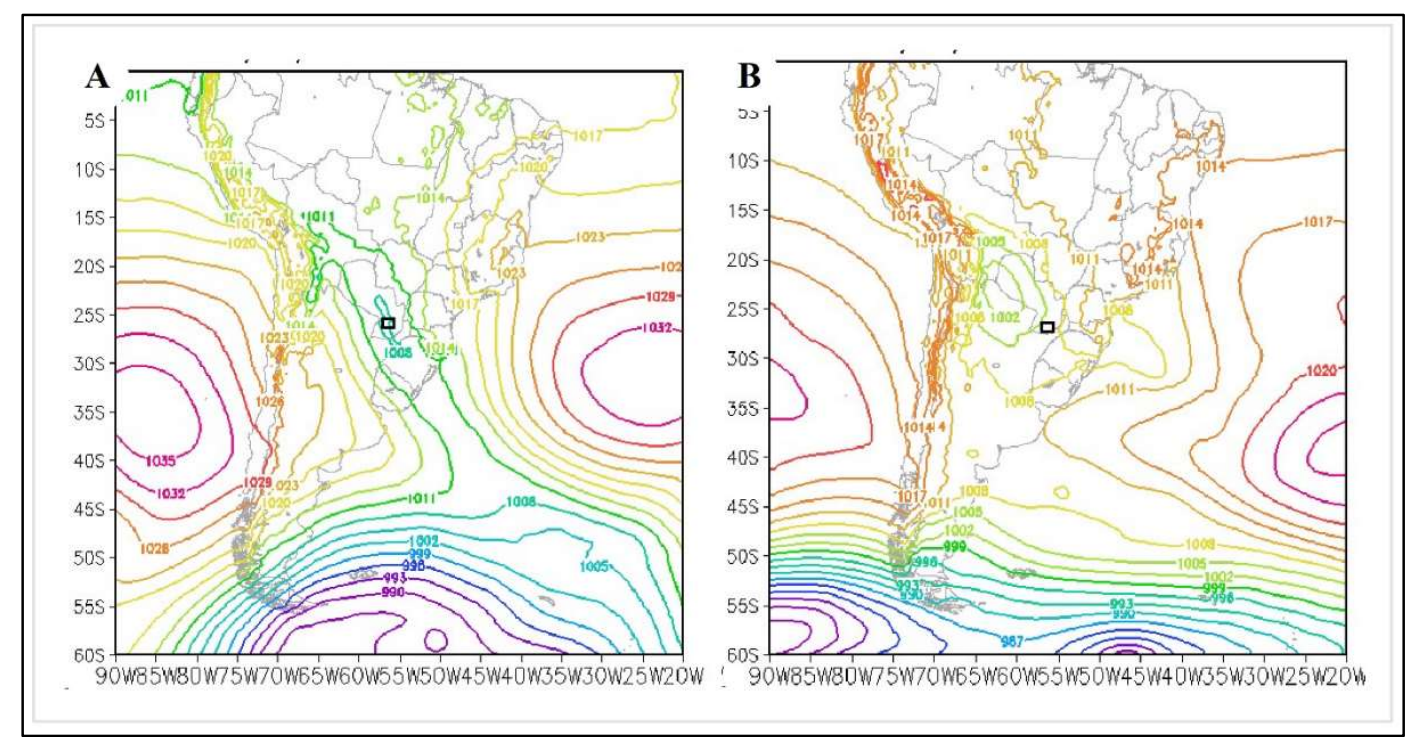

Figura 4: Campos medios diarios de pnmm en hPa (contorno en colores), en las localidades (A) Villarica 23/07/2014;

(B) Capitán Meza 26/01/2002. El rectángulo negro indica la localización de las áreas en estudio.

En la Figura 4 se nota sistemas de baja presión sobre las ciudades de Villarica y Capitán Meza. Estos sistemas indican condiciones de instabilidad en superficie, que asociados con la Baja del Chaco (BCH) (para el caso de verano, Figura 4B) pueden ser intensificados dinámicamente por vaguadas en altos niveles y térmicamente en bajos niveles por la escorrentía del viento de norte (Figura 7B-E y 8B-E) (SELUCHI et al., 2003; ESCOBAR et al., 2012). No obstante, para el caso de invierno (Figura 4A) no se percibe un centro de baja bien definido, sobre la ciudad existe una vaguada que antecede el anticiclón polar que esta sobre la Argentina.

En la Figura 5 se presentan los campos de espesor de la capa entre 500-1000hPa. En la Figuras 5A y 5D se observan en las normales las diferentes regiones de contraste térmico, con capas más cálidas para ambas ciudades en estudio. Así, en el día del evento de invierno (Figura 5B), se nota la presencia de una gran 
vaguada térmica, es decir, un sistema de gran amplitud, que cubre toda Argentina, lo que indica una atmósfera más delgada y, por lo tanto, más fría. Al este de esta vaguada térmica, hay una cuña térmica, que indica una capa cálida y, en consecuencia, un fuerte gradiente a barlovento de la región en estudio. Por lo tanto, Paraguay está bajo una atmósfera más cálida, pero muy cerca de la franja baroclínica. La vaguada y la cuña térmica se destacan en el campo de anomalía (Figura $5 \mathrm{C}$ ), donde se puede observar una mayor magnitud en la vaguada, lo que indica que es una masa polar intensa.

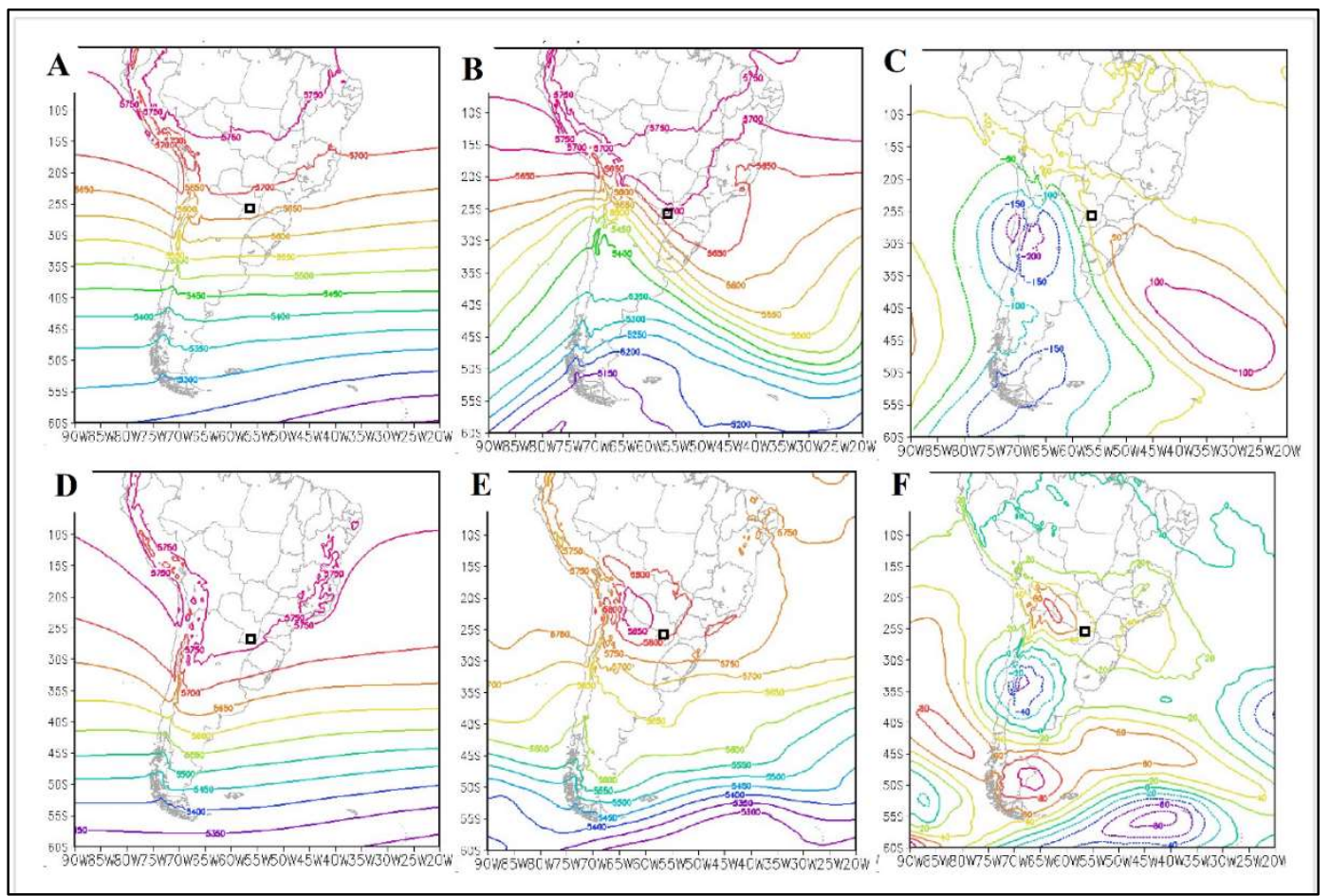

Figura 5: Campo medio de espesor (mgp) entre 500-1000hPa (columna a la izquierda); Campo de espesor en 500-

$1000 \mathrm{hPa}$ en el día del evento (columna central); Campo de anomalía de espesor en 500-1000hPa (columna a la derecha). (A, B, C) Villarica 23/07/2014; (D, E, F) Capitán Meza 26/01/2002. El rectángulo negro indica las áreas en estudio.

Para el caso de verano se verifica que Paraguay tiene una camada con espesura local máxima, indicando un ambiente cálido, más al sur, se observa una vaguada sobre los Andes (Figura 5E). El campo de anomalía (Figura 5F) indica que este máximo local tiene valores positivos próximos a la normal (Figura 5D). Al comparar este campo de anomalías con el del caso de julio, se observa que el caso de invierno presenta una anomalía con una magnitud mucho mayor, especialmente debido a la vaguada en el centro norte de Argentina.

En la Figura 6 se presenta el campo de la altura geopotencial en 500hPa. Para el caso de invierno, se observa la presencia de una vaguada de gran amplitud (destacada en el campo de anomalía en la Figura 6C) (Figura 6B) con eje al oeste de la región de estudio, indicando según la teoría cuasi-geostrófica, que el local de estudio está bajo una región de advección de vorticidad ciclónica, siendo una forzante dinámica contribuye para inestabilidad en superficie (HOLTON, 2004).

Para el caso de verano en la Figura 6E muestra la vaguada sobre Argentina, pero sin influencia aparente sobre la región en estudio, como lo demuestra el campo de anomalía (Figura 6F) cuyos valores cercanos a cero, representan un escenario de niveles promedio sin cambios importantes en relación con el 
patrón climático promedio (Figura 6D), bastante diferente al caso de invierno. Es decir, en el caso del verano no hubo influencia de la fuerza dinámica (vaguada preexistente en altos niveles).

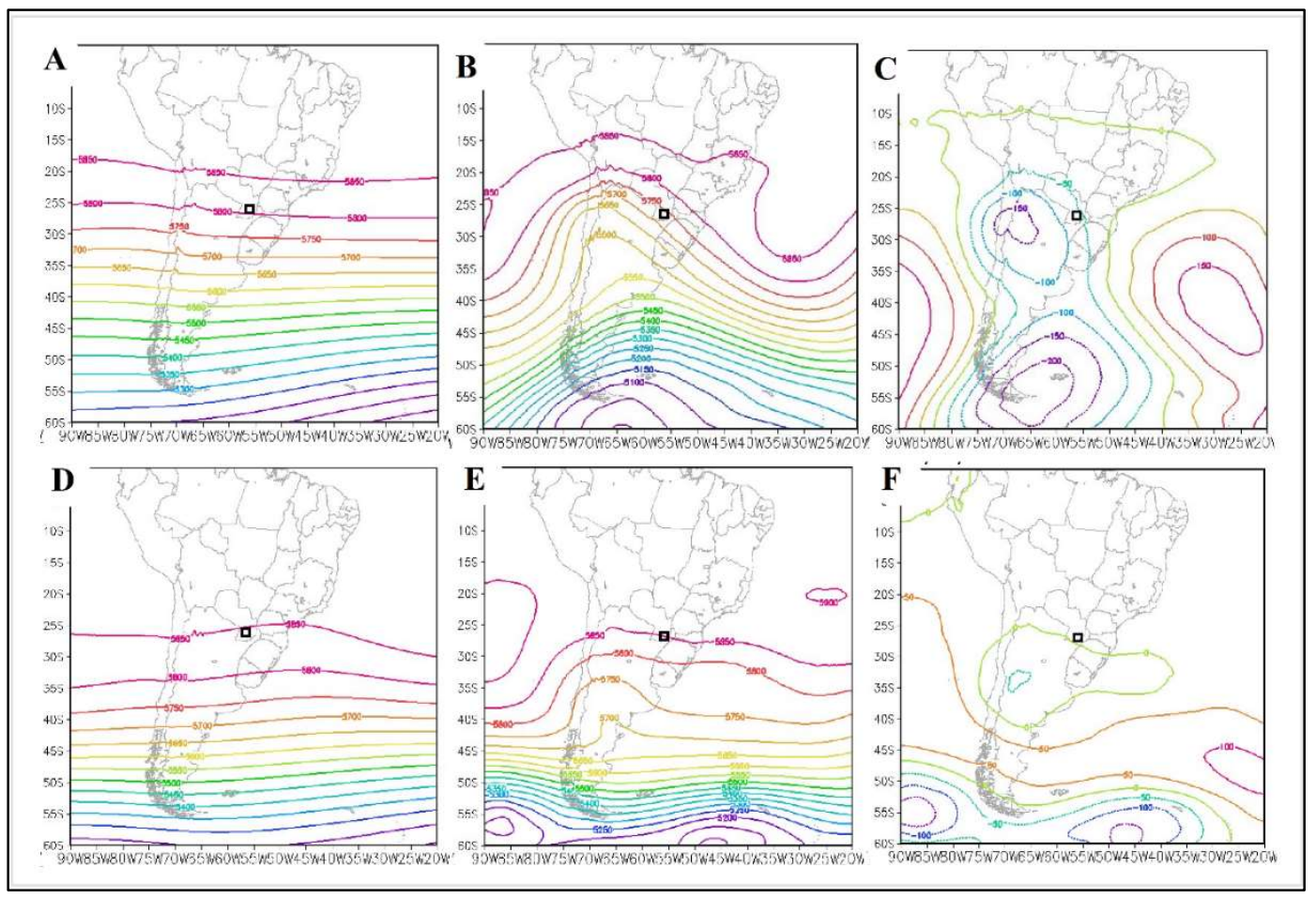

Figura 6: Campo medio de la altura geopotencial (mgp) en 500hPa (columna a la izquierda); Campo de altura geopotencial en $500 \mathrm{hPa}$ en el día del evento (columna central); Campo de anomalía de la altura geopotencial en 500hPa (columna a la derecha). (A, B, C) Villarica 23/07/2014; (D, E, F) Capitán Meza 26/01/2002. El rectángulo negro indica las áreas en estudio.

Se observa que la región de Villarica se encuentra bajo una gran difluencia en altos niveles (Figura 7B), un aspecto que se observa a menudo en casos de inestabilidad en superficie (SANDERS, 1993). La vaguada observada en 500hPa (Figura 6B) también se observa en $250 \mathrm{hPa}$ (Figura 7B). En relación a la Corriente en chorro subtropical, la región en estudio se encuentra en el sector polar de la salida (Figura 7A), lo que también puede estar asociado con inestabilidad en superficie, de acuerdo con el patrón de 4 celdas (SHAPIRO et al., 1981; MOORE et al., 1993). En el campo de anomalía (Figura 7C), se observa un ligero valor de magnitud negativa, normal en casos de difluencia.

Este campo de viento en $250 \mathrm{hPa}$ para el evento de verano muestra que Paraguay está bajo una circulación anticiclónica que, como lo indica el campo de espesura, está asociado con el calentamiento de la capa. Se observa el flujo ligeramente difluente sobre la región en estudio (Figura 7E), que separa la circulación anticiclónica y la vaguada sobre Argentina. Esta circulación anticiclónica es esperada, pero en menor magnitud, ya que surge en el campo de anomalías (con un centro sobre el sureste de Brasil) (Figura 7F). Es decir, la mayor diferencia de este caso con la normal climatológica (Figura 7D) es que el centro de la circulación ciclónica anómala se encuentra en el sureste de Brasil. A diferencia del invierno, la Corriente en chorro subtropical está al sur de la región en estudio.

Se observa, como esperado, la presencia de grandes vientos del norte al nivel de $850 \mathrm{hPa}$, lo que indica la presencia del Jet de bajos niveles (JBN) en Villarica (Figura 8B). La normal climatológica (Figura 8A) ya indica la presencia de escorrentía de norte en la región en esta época del año debido al anticiclón 
semipermanente del Atlántico Sur que en invierno adentra más al continente, sin embargo, a causa de la gran magnitud de la escorrentía en el día del evento (Figura 8B), el campo de anomalía muestra que esta es una escorrentía excepcional (Figura 8C). Particularmente, este JBN se encuentra a sotavento de la vaguada frontal, intensificando la advección cálida pre frontal.

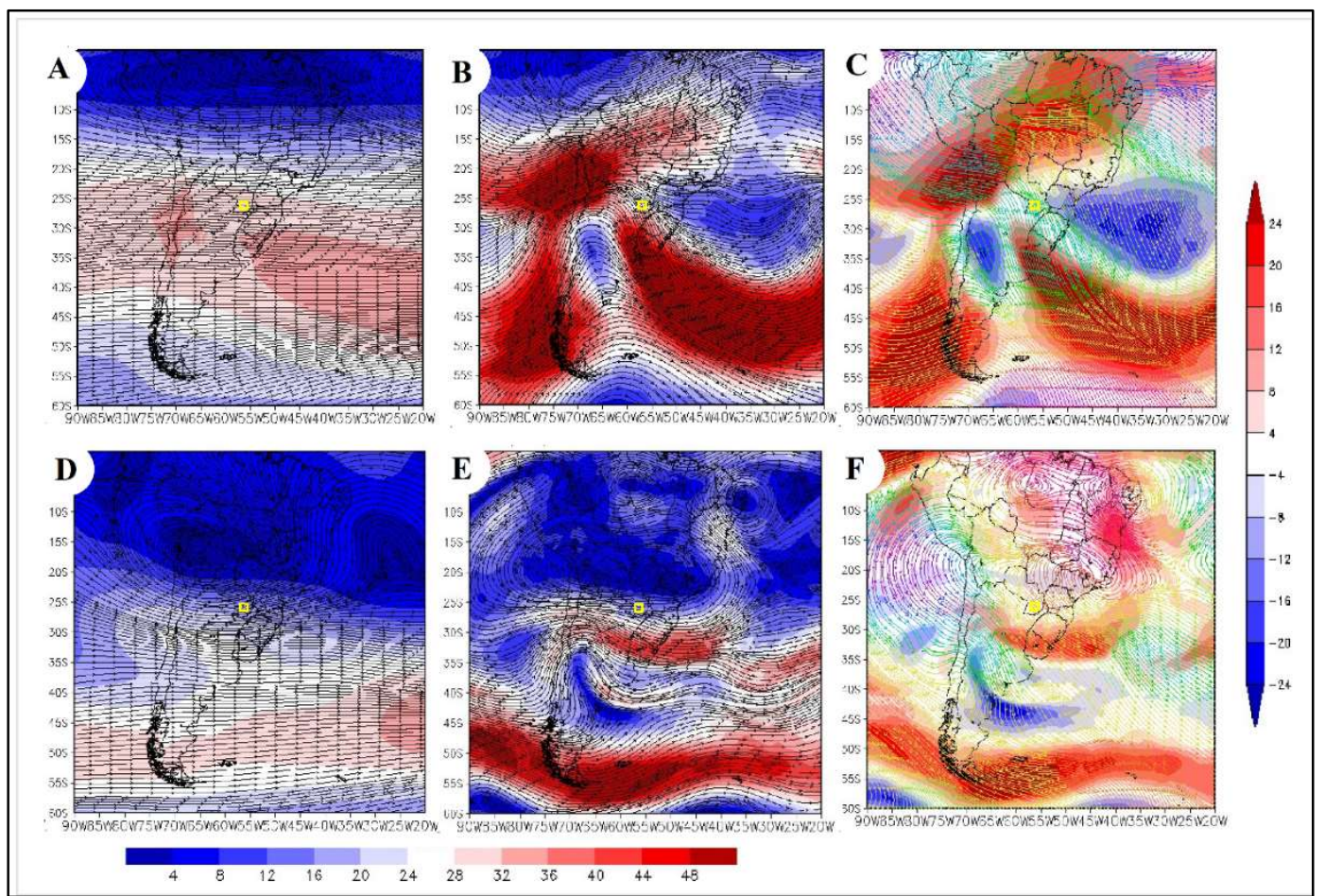

Figura 7: Campo medio de línea de corriente (contorno) y magnitud del viento (sombreado) en $\mathrm{m} \mathrm{s}^{-1}$ en $250 \mathrm{hPa}$ (columna a la izquierda); Campo de línea de corriente y magnitud del viento en el día del evento (columna central);

Campo de anomalía de la línea de corriente y magnitud del viento (columna a la derecha). (A, B, C) Villarica 23/07/2014; (D, E, F) Capitán Meza 26/01/2002. El rectángulo amarillo indica las áreas en estudio.

Como en el caso del invierno, este campo muestra una gran magnitud de escorrentía septentrional sobre la región, lo que indica la presencia de JBN (Figura 8E). La mayor diferencia para el caso de invierno es que en verano el JBN se limita a la región de estudio, mientras que en invierno el flujo avanza a latitudes mayores.

Estudios llevados a cabo por Virji (1981), Paegle et al. (1987) y Salio et al. (2007) explican que JBN Sul Americano desempeña un papel importante en el clima, resultante del transporte calor y la humedad de la región Amazónica hacia la Cuenca del Río de la Plata en las planicies centrales del sudeste de América del Sur, generando condiciones ambientales ideales para el inicio de convección y crecimiento en Sistemas Convectivos de Mesoescala (SCMs), estando posicionado al este de la Codillera de los Andes, atraviesa el continente entre 15 y los 30`S (SAULO et al., 2000; MARENGO et al., 2004). La configuración del sistema a $250 \mathrm{hPa}$, actuando con difluencia de la Corriente en chorro subtropical, asociado al JBN en $850 \mathrm{hPa}$, sobre las localidades en estudio propicia los eventos convectivos intensos.

En este contexto, resultados encontrados por Riquetti et al. (2018) destacan la influencia del JBN y de la Corriente en chorro subtropical en el evento de precipitación extrema ocurrido en septiembre de 2015 en la ciudad de Foz do Iguaçu-Brasil, ocasionando severas pérdidas económicas, ambientales y sociales para la población en la región. En este sentido, Seluchi et al. (2000), Vera et al. (2006), entre otros, destacan que 
el principal agente de transporte de calor y humedad es el JBN, relacionados a la formación de sistemas convectivos de Mesoescala sobre la región del Chaco paraguayo.

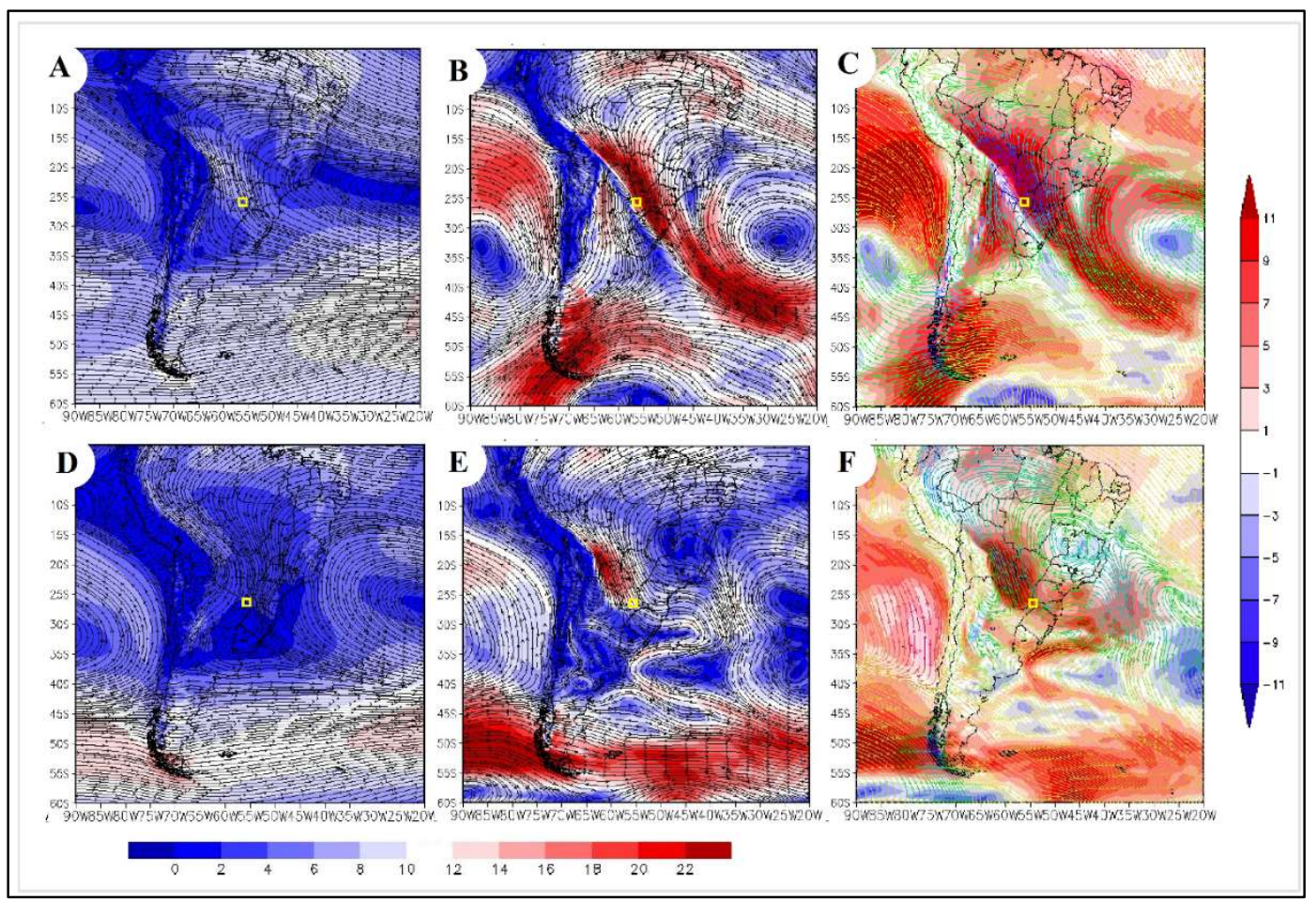

Figura 8: Campo medio de línea de corriente (contorno) y magnitud del viento (sombreado) en $\mathrm{m} \mathrm{s}^{-1}$ en $850 \mathrm{hPa}$ (columna a la izquierda); Campo de línea de corriente y magnitud del viento en $\mathrm{m} \mathrm{s}^{-1}$ en el día del evento (columna central); Campo de anomalía de la línea de corriente y magnitud del viento en $\mathrm{m} \mathrm{s}^{-1}$ (columna a la derecha). (A, B, C)

Villarica 23/07/2014; (D, E, F) Capitán Meza 26/01/2002. El rectángulo amarillo indica las áreas en estudio.

En la Figura 9 se muestra las imágenes satélites del canal infrarrojo resaltando para las localidades en estudio los núcleos de mayor convección. Debido a que son eventos convectivos con duración de horas, se observa en las imágenes la hora de máximo rendimiento del evento extremo e intenso de precipitación.
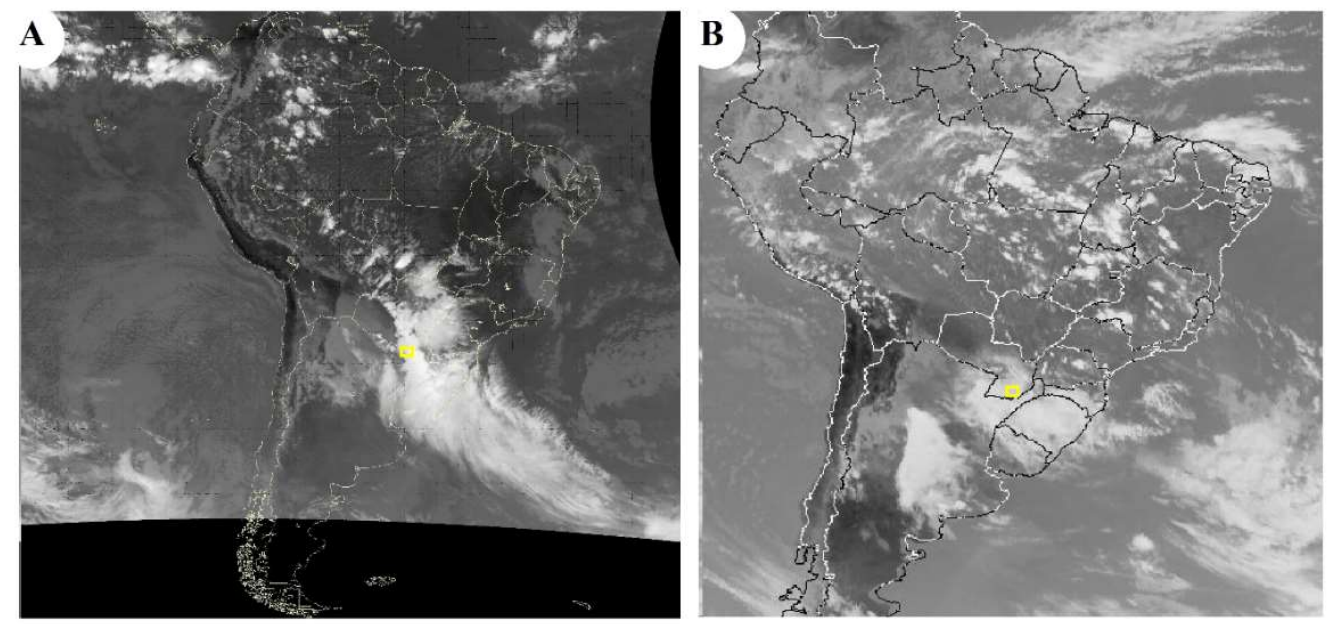

Figura 9: Imágenes del satélite GOES-13 y GOES-8 del canal infrarrojo para los casos de eventos intensos. A) Villarica (23/07/2014 18:30UTC); B) Capitán Meza (26/01/2002 18:10UTC). El rectángulo amarillo indica las áreas en estudio.

\section{CONCLUSIONES}

Este trabajo analizó los casos de precipitación intensa en Paraguay durante el periodo de 1999-2019. 
De esta forma, se concluye que en la región Occidental del Paraguay se encontró predominancia de eventos de precipitación intensa (p99) en primavera y verano, sin ningún caso para el invierno. Sin embargo, para la región Oriental el comportamiento de los eventos p99 es más homogéneo durante el año, con número de ocurrencias más pronunciado en el centro de la región Oriental. En relación al comportamiento de la precipitación intensa para Villarica (23/07/2014) y Capitán Meza (26/01/2002) las tendencias analizadas son positivas pero no significativas estadísticamente, en tanto, se destaca la discrepancia existente entre las regiones del país en términos de cantidad de eventos intensos anual y por estación del año.

Según analizado, para los estudios de casos se comprobó que el comportamiento de las variables en estudio que causaron los eventos intensos en las dos ciudades estaba asociado a las forzantes térmicas en bajos niveles (especialmente el JBN) y forzamientos dinámicos provenientes de altos niveles, coincidiendo con la literatura especializada. El caso de invierno, más raro, presentó anomalías más intensas, así también la acción de forzantes dinámicas de altos niveles como la presencia de una vaguada al oeste que intensificó la instabilidad baroclínica en superficie.

AGRADECIMIENTOS: A la Coordenação de Aperfeiçoamento de Pessoal de Nível Superior - Brasil (CAPES) Código de Financiamiento 001. A la Dirección Nacional de Meteorología - Paraguay (DINAC) por la disponibilidad de los datos de las series históricas de precipitación.

\section{REFERENCIAS}

ALEXANDER, L. V.; ZHANG, X.; PETERSON, T. C.; CAESAR, J.; GLEASON, B.; KLEIN TANK, A. M. G.; HAYLOCK, M.; COLLINS, D.; TREWIN, B.; RAHIMZADEH, F.; TAGIPOUR, A.; RUPA KUMAR, K.; REVADEKAR, J.; GRIFFITHS, G.; VINCENT, L.; STEPHENSON, D. B.; BURN, J.; AGUILAR, E.; BRUNET, M.; TAYLOR, M.; NEW, M.; ZHAI, P.; RUSTICUCCI, M.; VAZQUEZAGUIRRE, J. L.. Global observed changes in daily climate extremes of temperature and precipitation. Journal of Geophysical Research, v.111, 2006. DOI: http://doi.org/10.1029/2005JD006290

BIDEGAIN, M.; PASTÉN, M.; NAGY, G. J.; CORONEL, G.; FERRER, J.; ARANA, I.. Tendencias recientes de las precipitaciones e impactos asociados con ENSO en la cuenca del Río de la Plata. Paraquaria Natural, v.5, p.8-18, 2017.

BREUER, N. E.; OREGGIONI, F.; BÁEZ, J.. Percepción y observación de las variaciones en el régimen pluviométrico en Itapúa y Alto Paraná. Paraquaria Natural, v.5, n.2, p.3744, 2017.

ESCOBAR, G. C. J.; SELUCHI, M. E.. Classificação sinótica dos campos de pressão atmosférica na América do Sul e sua relação com as baixas do Chaco e do noroeste argentino. Revista Brasileira de Meteorologia, v.27, p.365-375, 2012. DOI: $\underline{\text { http://doi.org/10.1590/S0102-77862012000300011 }}$

GRIMM, A. M.. The El Niño impact on the summer monsoon in Brazil: regional processes versus remote influences.

Journal of Climate, v.16, n.2, p.263-280, 2003. DOI:

http://doi.org/10.1175/1520-

0442(2003)016\%3C0263:TENIOT\%3E2.0.CO;2
GRIMM, A. M.. Interannual climate variability in South America: impacts on seasonal precipitation, extreme events and possible effects of climate change. Stochastic Environmental Research and Risk Assessment, v.25, n.4, p.537-554, 2011. DOI: http://doi.org/10.1007/s00477-010$\underline{0420-1}$

HAYLOCK, M. R.; PETERSON, T. C.; ALVES, L. M.; AMBRIZZI, T.; ANUNCIAÇÃO, Y. M. T.; BAEZ, J.; BARROS, V. R.; BERLATO, M. A.; BIDEGAIN, M.; CORONEL, G.; GARCIA, V. J.; GRIMM, A. M.; KAROLY, D.; MARENGO, J. A.; MARINO, M. B.; MONCUNILL, D. F.; NECHET, D.; QUINTANA, J.; REBELLO, E.; RUSTICUCCI, M.; SANTOS, J. L.; TREBEJO, I.; VINCENT, L. A.. Trends in total and extreme South American rainfall 19602000 and links with sea surface temperature. Journal of Climate, v.19, p.1490-1512, 2006. DOI: http://doi.org/10.1175/JCLI3695.1

HERSBACH, H.; BELL, B.; BERRISFORD, P.; HORÁNYI, A.; SABATER, J. M.; NICOLAS, J.; RADU, R.; SCHEPERS, D.; SIMMONS, A.; SOCI, C.; DEE, D.. Global reanalysis: goodbye ERA-Interim, hello ERA5. ECMWF Newsletter, v.159, p.1724, 2019. DOI: http://dx.doi.org/10.21957/vf291hehd7

HOLTON, J. R.. Introduction to Dynamic Meteorology. Amsterdam: Elsevier, 2004.

LIEBMANN, B.; VERA, C. S.; CARVALHO, L. M. V.; CAMILLONI, I. A.; HOERLING, M. P.; ALLURED, D.; BARROS, V. R.; BÁEZ, J.; BIDEGAIN, M.. An observed trend in central South American Precipitation. Journal of Climate, v.17, n.22, p.4357-4367, 2004. DOI: http://doi.org/10.1175/3205.1 
MARENGO, J.; SOARES W.; SAULO, C.; NICOLINI, M. Climatology of Low-Level Jet East of the Andes as derived from the NCEP-NCAR reanalyses: Characteristics and Temporal Variability. Journal of Climate, v.17, p.2261-2280, 2004. DOI: http://doi.org/10.1175/15200442(2004)017\%3C2261:COTLJE\%3E2.0.CO;2

MENDONÇA, M.; ROMERO, H.; OPAZO, D.. Análise multiescalar para a compreensão de causas e consequências da variabilidade climática na América do Sul. In: DA SILVA, C. A.; FIALHO, E. S.; STEINKE, E. T.. Experimentos em Climatologia Geográfica. Dourados: Universidade Federal Grande Dourados, 2014. p.271-289.

MOORE, J. T.; VANKNOWE, G. E.. The Effect of Jet-Streak Curvature on Kinematic Fields. Monthly Weather Review, v.120, p.2429-2441, 1993. DOI:

http://doi.org/10.1175/15200493(1992)120\%3C2429:TEOJSC\%3E2.0.CO;2

OLIVEIRA, A. S.. Interações entre sistemas frontais na América do Sul e a convecção na Amazônia. Dissertação (Mestrado em Meteorologia) - Instituto Nacional de Pesquisas Espaciais, São José dos Campos, 1986.

PAEGLE, J.; ZHANG, C.. Atmospheric response to tropical thermal forcing in real data integrations. Monthly Weather Review, v.115, p.2975-2995, 1987. DOI:

http://doi.org/10.1175/1520-

0493(1987)115\%3C2975:ARTTTF\%3E2.0.CO;2

PASTÉN, A. M.; ARMOA, J.; BENÍTEZ, M.. Análisis de eventos meteorológicos intensos en el Paraguay. Informe Final, Proyecto: FP/08/07. San Lorenzo: Ciudad Universitaria, 2007.

PEEL, M. C.; FINLAYSON, B. L.; MCMAHON, T. A.. Updated world map of the Köppen-Geiger climate classification. Hydrology and Earth System Sciences, v.11, p.1633-1644, 2007. DOI: http://doi.org/10.5194/hess-11-1633-2007

REBOITA, M. S.; GAN, M. A.; ROCHA, R. P.; AMBRIZZI, T.. Regimes de precipitação na América do Sul: uma revisão bibliográfica. Revista Brasileira de Meteorologia, v.25, n.2, p.185-204, 2010. DOI: http://doi.org/10.1590/S0102$\underline{77862010000200004}$

REBOITA, M.; KRUSCHE, N.; AMBRIZZI, T.; ROCHA, R.. Entendendo o tempo e o clima na América do Sul. Terra e Didática, v.8, p.34-50, 2012. DOI:

http://doi.org/10.20396/td.v8i1.8637425

RIQUETTI, N. B.; DORNELES, V. R.; NUNES, A. B.. Estudo do caso de precipitação intensa sobre Foz do Iguaçu-PR em setembro de 2015. Revista Brasileira de Climatologia, v.22, p.589-606, 2018. DOI:

http://dx.doi.org/10.5380/abclima.v22i 0.58304

SALIO, P.; NICOLINI, M.; ZIPSER, E. J.. Mesoscale convective systems over South America and their relationship with the South American low-level jet. Monthly Weather Review, v.135, p.1290-1309, 2007. DOI:

http://doi.org/10.1175/MWR3305.1

SANDERS, F.. Upper-Level Geostrophic Diffluence and
Deepening of Surface Lows. Weather and Forecasting, v.8, p.338-344, 1993. DOI: http://doi.org/10.1175/15200434(1993)008\%3C0339:ULGDAD\%3E2.0.CO;2

SATYAMURTY, P.; NOBRE, C. A.; SILVA DIAS, P. L.. South America. In: KAROLY, D. J.; VICENT, D. G.. Meteorology of the Southern Hemisphere. American Meteorological Society, Meteorological Monographs, 1998. p.119-139.

SAULO, C.; NICOLINI, M.; CHOU, S.. Model characterization of the South American low-level flow during the 1997-98 spring-summer season. Climate Dynamics, v.16, p.867-881, 2000. DOI: http://doi.org/10.1007/s003820000085

SELUCHI, M. E.; MARENGO, J. A.. Tropical-midlatitude exchange of air masses during summer and winter in South America: Climatic aspects and extreme events. International Journal of Climatology, v.20, p.1167-1190, 2000. DOI: http://doi.org/10.1002/10970088(200008)20:10\%3C1167::AID-JOC526\%3E3.0.CO;2-T

SELUCHI, M. E.; SAULO, A. C.; NICOLINI, M.; SATYAMURTY, P.. The Northwestern Argentinean Low: A study of two typical events. Monthly Weather Review, v.131, p.23612378, 2003. DOI: http://doi.org/10.1175/15200493(2003)131\%3C2361:TNALAS\%3E2.0.CO;2

SELUCHI, M. E.; GARREAUD, R. D.. Campos médios e processos físicos associados ao ciclo de vida da Baixa do Chaco. Revista Brasileira de Meteorologia, v.27, n.4, p.447462, 2012a. DOI: http://doi.org/10.1590/S010277862012000400008

SELUCHI, M. E.; SAULO, A. C.. Baixa do noroeste argentino e Baixa do Chaco: Características, diferenças e semelhanças. Revista Brasileira de Meteorologia, v.27, n.1, p.49-60, 2012b. DOI: http://doi.org/10.1590/S0102$\underline{77862012000100006}$

SHAPIRO, M. A.; KENNEDY, P. J.. Research aircraft measurements of jet stream geostrophic and ageostrophic winds. Journal of the Atmospheric Sciences, v.38, p.26422652, 1981. DOI: http://doi.org/10.1175/15200469(1981)038\%3C2642:RAMOJS\%3E2.0.CO;2

VASQUES, A. C.. Características de precipitação sobre a América do Sul provenientes de diferentes fontes de dados com ênfase no Brasil. Dissertação (Mestrado em Meteorologia) - Instituto Nacional de Pesquisas Espaciais, São José dos Campos, 2007.

VERA, C. S.; BAEZ, J.; DOUGLAS, M.; EMMANUEL, C. B.; MARENGO, J. A.; MEITIN, J.; NICOLINI, M.; NOGUES-PEAGLE, J.; PEAGLE, J.; PENALBA, O.; SALIO, P.; SAULO, C.; SILVA DIAS, M. A.; DIAS, P. S.; ZIPSER, E.. The South American low-Level Jet Experiment. Bulletin of American Meteorological Society, v.87, p.63-77, 2006. DOI: http://doi.org/10.1175/BAMS-87-1-63

VIRJI, H.. A preliminary study of summertime tropospheric circulation patterns over South America estimated from clouds winds. Monthly Weather Review, v.109, p.599-610, 1981. DOI: http://doi.org/10.1175/15200493(1981)109\%3C0599:APSOST\%3E2.0.CO;2

A CBPC - Companhia Brasileira de Produção Científica (CNPJ: 11.221.422/0001-03) detém os direitos materiais desta publicação. Os direitos referem-se à publicação do trabalho em qualquer parte do mundo, incluindo os direitos às renovações, expansões e disseminações da contribuição, bem como outros direitos subsidiários. Todos os trabalhos publicados eletronicamente poderão posteriormente ser publicados em coletâneas impressas sob coordenação da Sustenere Publishing, da Companhia Brasileira de Produção Científica e seus parceiros autorizados. Os (as) autores (as) preservam os direitos autorais, mas não têm permissão para a publicação da contribuição em outro meio, impresso ou digital, em português ou em tradução. 